\section{Actividad física, obesidad y autoestima en escolares chilenos: Análisis mediante ecuaciones estructurales}

\author{
FÉLIX ZURITA-ORTEGA ${ }^{1, \mathrm{a},}$, MANUEL CASTRO-SÁNCHEZ ${ }^{2, \mathrm{a}}$, \\ SONIA RODRÍGUEZ-FERNÁNDEZ ${ }^{3, \mathrm{a}}$, CRISTIAN COFRÉ-BOLADÓS ${ }^{4,5, \mathrm{a}}$, \\ RAMÓN CHACÓN-CUBEROS ${ }^{2}$, ASUNCIÓN MARTIINEZ-MARTIINEZ ${ }^{3, a}$, \\ JOSÉ JOAQUÍN MUROS-MOLINA ${ }^{6, a}$
}

\section{Physical activity, obesity and self-esteem in chilean schoolchildren}

Background: Obesity is a worldwide epidemic disease and a problem for the Chilean society. Aim: To analyze the relationship between physical condition, body mass index (BMI), level of physical activity and self-esteem. Material ad Methods: Questionnaires to assess self-esteem (Rosemberg scale) and levels of physical activity (Physical Activity Questionnaire for older Children, PAQ-C) were answered by 515 children aged $10.5 \pm 0.5$ years from 27 schools of Santiago de Chile. BMI was calculated. Course-Navette test was carried out, vertical jump and hand dynamometry were measured. For statistical analysis, structural equations were used. Results: An acceptable goodness of fit for the models was found. There was a positive relationship between BMI and hand dynamometry, as well as a negative relationship between BMI and maximal oxygen consumption, jumping ability, physical activity and self-esteem. Finally, self-esteem was positively related to physical activity engagement. Conclusions: In these children, self-esteem was related to physical activity variables.

(Rev Med Chile 2017; 145: 327-334)

Key words: Body Mass Index; Child; Chile; Physical Fitness; Self Concept.

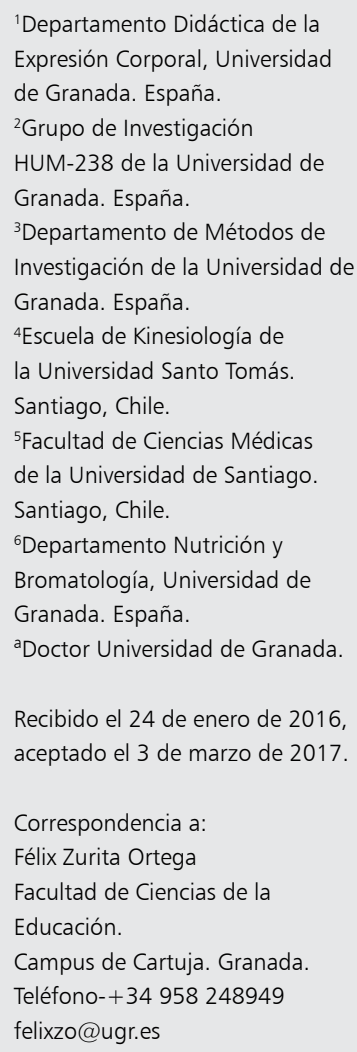

E 1 Ministerio de Salud de Chile a través de su Estrategia Nacional de Salud, trazada $\checkmark$ para la década 2011-2020, se ha propuesto potenciar acciones de promoción de salud. Esta estrategia tiene como objetivo reducir los factores de riesgo asociados al consumo de tabaco y alcohol, sobrepeso, sedentarismo, conducta sexual y consumo de drogas ilícitas ${ }^{1}$. En este sentido, varios estudios hacen énfasis en la importancia de intervenir desde edades tempranas en estos aspectos, no sólo reduciendo hábitos no saludables, sino disminuyendo los costes asociados a las patologías crónicas modernas desde los sistemas públicos $^{2-4}$.
Algunos de los principales organismos de salud pública a nivel mundial han establecido directrices sobre hábitos saludables, siendo la Actividad Física (AF) fundamental, definiéndose en cuanto al tipo, cantidad e intensidad necesaria para asegurar un estado adecuado de salud ${ }^{5,6}$. De este modo, se destaca la importancia del desarrollo de diversos hábitos saludables para la promoción de la salud, como seguir patrones alimentarios adecuados, práctica físico-deportiva y ausencia de consumo de sustancias nocivas ${ }^{1-3,7}$.

La condición física, determinada a partir de las capacidades del organismo para obtener un buen rendimiento en su funcionamiento, puede ayudar 
a determinar el estado de salud de un sujeto. La capacidad aeróbica es una de las cualidades más importantes, dado que se asocia a la capacidad del organismo para funcionar eficientemente y de realizar esfuerzos de forma sostenida ${ }^{8,9}$. En la misma línea, el nivel de fuerza muscular, entendida como otra cualidad determinante en el estado de salud, representa un factor de riesgo que se relaciona con enfermedades de tipo cardiovascular ${ }^{10}$. Asimismo, el nivel de acondicionamiento muscular en la infancia tardía y la adolescencia parece perdurar en la edad adulta ${ }^{11}$.

La autoestima es un factor crítico que afecta al ajuste psicosocial del individuo y que puede relacionarse con estados de salud concretos. Niveles bajos en los jóvenes se han asociado con síntomas psicopatológicos como reacciones de ansiedad ${ }^{12}$, depresión, desesperanza y tendencias suicidas ${ }^{13}$, demoras innecesarias en la realización de tareas y son comúnmente observados en aquellos que presentan conductas agresivas ${ }^{14}$, conductas antisociales ${ }^{15} \mathrm{o}$ violencia escolar ${ }^{16}$. También es fundamental analizar que los niños y jóvenes con baja autoestima se caracterizan por presentar una peor salud física, encontrando que los sujetos que llegan a la adolescencia con baja autoestima tienen un riesgo mayor de presentar hábitos no saludables y una salud mental más pobre ${ }^{17}$.

Se ha hecho hincapié en que la transición epidemiológica de Chile ha favorecido el exceso de peso en todos los grupos de edad ${ }^{18,19}$. Este es un punto importante considerando que los factores culturales y el comportamiento del modelo de alimentación parecen ser muy relevantes tanto en la conformación de obesidad, como en su tratamiento $^{20}$. Los términos abordados en este documento discurren por un eje común, definiendo como se asocian las capacidades físicas con la obesidad, autoestima y practica de AF.

En relación a lo planteado, este estudio tiene el objetivo general de analizar las relaciones existentes entre una serie de variables físicas y psicológicas, desglosándose en dos objetivos específicos: a) desarrollar un modelo explicativo del nivel de obesidad y práctica de AF en escolares chilenos y que integre otras variables relacionadas con la condición física y la práctica de AF; b) analizar las relaciones existentes entre las variables físico-motrices y el nivel de obesidad, autoestima y práctica de $\mathrm{AF}$, partiendo del modelo explicativo desarrollado.

\section{Material y Método}

\section{Diseño y participantes}

Se realizó una investigación de carácter descriptivo, transversal y exploratorio. El universo total del estudio $(n=2.568)$ estuvo representado por los escolares de cuarto básico $(\mathrm{n}=1.272)$ y quinto básico $(\mathrm{n}=1.296)$ de 27 centros educativos de Santiago de Chile (censo realizado en diciembre de 2014 e información provista por el departamento de Educación Física de la Asociación Cristiana de Jóvenes de Santiago). Para establecer una muestra representativa (error muestral del 0,04; IC $=95,5 \%$ ) se realizaron técnicas de estratificación, proporcionalidad y aleatorización. Se empleó un muestreo aleatorio estratificado con afijación proporcional, con el fin asegurar una representación homogénea de la muestra. Los estratos considerados fueron el curso (cuarto y quinto básico) y el sexo (varones y mujeres). El estrato generado por cuarto básico estuvo constituido por 1.272 sujetos $(49,53 \%)$. Una vez fijada la proporción a seguir en la selección de participantes, se seleccionaron 255 escolares mediante muestreo aleatorio simple a partir del listado de alumnos matriculados en los 27 centros educativos. Se estableció como criterio de selección la elección de un máximo de 19 participantes por colegio con el fin de asegurar una adecuada representación de cada centro. El estrato definido por quinto básico estuvo constituido por 1.296 escolares $(50,47 \%)$. Se realizó el mismo procedimiento para asegurar su representación, seleccionando 260 escolares de quinto básico. En la selección de participantes para los estratos por curso se guardó la proporción del sexo fijada por el total. Dado que el universo total estuvo constituido por $50,48 \%$ de varones $(\mathrm{n}=1.296)$ y $49,52 \%$ de mujeres $(\mathrm{n}=1.272)$, se estableció como afijación proporcional una representación de 260 hombres y 255 damas. Finalmente, la muestra estuvo compuesta por 515 niños y niñas en edad escolar con una edad comprendida entre los 10 y 11 años $(M=10,55$ años; DT = 0,498).

\section{Instrumentos}

Para la evaluación de las variables relacionadas con la condición física se empleó el test Course Navette para determinar el $\mathrm{VO}_{2} \mathrm{Max}$ (potencia aeróbica); dinamometría manual para medir la fuerza de prensión manual en brazo dominante; 
el test de Salto Vertical con el fin de determinar la fuerza explosiva del tren inferior mediante distancia máxima alcanzada; y las horas de AF semanales a través de una hoja de auto-registro.

Para establecer el nivel de autoestima de los participantes se empleó el test de Rosenberg ${ }^{21}$. Este cuestionario consta de 10 items que son puntuados mediante una escala Likert de 4 opciones, donde $1=$ "Muy en desacuerdo" y 4 = "Muy de acuerdo". Los ítems 1, 3, 4, 7 y 10 están redactados de forma positiva, y los ítems 2, 5, 6, 8 y 9 están redactados en forma negativa. Finalmente, se realiza una sumatoria que determina el nivel de autoestima. Este instrumento ha sido adaptado al español ${ }^{22}$.

El Índice de Masa Corporal (IMC) fue determinado a través del peso y la altura (masa/talla ${ }^{2}$ ). Para la medición del peso de los escolares se empleó una báscula tipo SECA 376, mientras que para la talla se utilizó un estadiómetro SECA 217.

El nivel de AF se valoró a través del cuestionario Physical Activity Questionnaire for older Children (PAQ-C), en su versión validada al español ${ }^{23}$. El cuestionario valora la práctica de AF realizada en los últimos 7 días a través de 10 preguntas sobre su tipo y frecuencia. De las respuestas se obtiene una puntuación que oscila entre 1 y 5 , indicando una mayor puntuación una mayor práctica de práctica de AF.

\section{Procedimiento}

Para la realización de este trabajo de investigación se han seguido las normas éticas de la declaración de Helsinki de 1975. Asimismo, se contó con el consentimiento informado de los responsables legales de los escolares, previamente informados de la naturaleza del estudio. En el proceso de aplicación de los instrumentos se contó con la presencia de los investigadores con el fin de asegurar una correcta aplicación de los mismos. Los escolares completaron los cuestionarios anónimamente, respetando su derecho de confidencialidad. Tuvieron que excluirse un total de 18 participantes que no completaron debidamente los cuestionarios o que se negaron a participar. Finalmente, se les señaló a los responsables legales que estuviesen interesados la posterior remisión de los datos una vez analizados.

\section{Análisis de los datos}

Para analizar las relaciones existentes entre las variables se realizó un modelo de ecuaciones estructurales con el fin de definir las relaciones entre los diferentes constructos implicados en el estudio, utilizando el programa AMOS 21. Para la estimación de los estadísticos descriptivos básicos se empleó el software SPSS 22.0.

\section{Resultados}

Con la finalidad de dar cumplimiento a los objetivos planteados, se realizó un modelo de ecuaciones estructurales con el fin de determinar las asociaciones entre los diferentes constructos implicados en el estudio. La especificación teórica del modelo, como se aprecia en la Figura 1, permite desarrollar relaciones entre las variables, de manera que unas variables se relacionen con otras variables que, a su vez, se asociarán con otras, creando concatenaciones de variables. Este tipo de modelos en particular también se denominan modelos de análisis de rutas (path analysis).

En nuestro modelo, las variables exógenas coindicen con las variables predictoras, es decir: Potencia aeróbica ( $\mathrm{VO}_{2} \mathrm{Max}$ ); salto (medido a través de la fuerza explosiva del tren inferior); y Dinamometría manual (establecida a través de la fuerza de prensión manual). Las variables endógenas son: el Índice de Masa Corporal (IMC); horas de dedicación a la AF (HorasAF); nivel de AF (NIVELPAQC); y por último, la autoestima.

Como ya hemos mencionado, nuestro modelo contiene variables observables, excepto los errores, que son variables latentes. Los términos de error asociados a las variables endógenas representan el error de predicción. Las relaciones bidireccionales entre las variables predictoras establecen la covariación entre las mismas. Por tanto, el modelo plantea la existencia de tres variables predictoras ( $\mathrm{VO}_{2} \mathrm{Max}$, Salto y Dinamometría manual) que influyen en el IMC. A su vez, esta última influye de manera directa en el nivel de AF (NIVELPAQC), las horas de AF (HorasAF) y la autoestima. Además, se establece la influencia directa entre el nivel de AF (NIVELPAQC) y la autoestima, y de manera indirecta entre ambas.

Interpretando la magnitud y el signo de los parámetros estimados (Figura 2), los resultados muestran que las variables predictoras $\mathrm{VO}_{2} \mathrm{Max}$ y Salto, se relacionan negativamente con el Índice de Masa Corporal (IMC), de manera que un mayor IMC se asociará a un menor $\mathrm{VO}_{2} \mathrm{Max}$ y una 

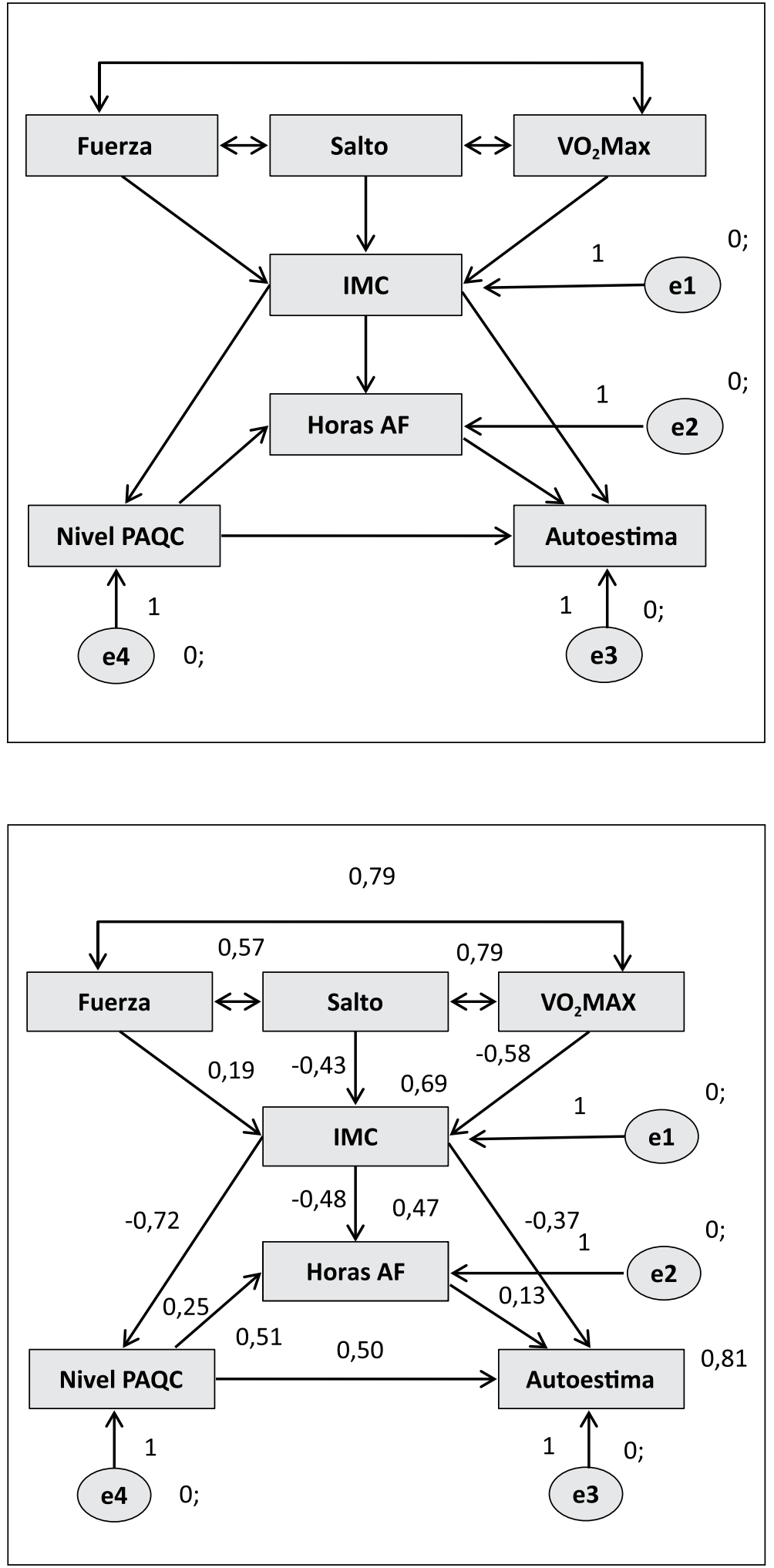

Figura 1. Modelo de rutas. $\mathrm{VO}_{2} \mathrm{Max}$ (Potencia Aeróbica); Salto (Test de Salto Vertical); Fuerza (Fuerza según dinamometría manual); IMC (Índice de Masa Corporal); Horas AF (Horas diarias de actividad físico-deportiva); NIVEL PAQC (Nivel de Actividad Física semanal); y Autoestima (Nivel de autoestima mediante el Test de Rosenberg).
Figura 2. Modelo de relaciones estructurales. $\mathrm{VO}_{2} \mathrm{Max}$ (Potencia Aeróbica); Salto (Test de Salto Vertical); Fuerza (Fuerza según dinamometría manual); IMC (Índice de Masa Corporal); Horas AF (Horas diarias de actividad físico-deportiva); NIVEL PAQC (Nivel de Actividad Física semanal); y Autoestima (Nivel de autoestima mediante el Test de Rosenberg). 
Tabla 1. Pesos de regresión y pesos estandarizados de regresión del modelo

\begin{tabular}{|c|c|c|c|c|c|c|c|}
\hline \multicolumn{3}{|c|}{ Relación entre variables } & \multicolumn{4}{|c|}{ PR } & \multirow{2}{*}{$\begin{array}{c}\text { PER } \\
\text { Estimaciones } \\
0,185\end{array}$} \\
\hline IMC & $<--$ & Fuerza (DINA) & 0,087 & 0,019 & 4,611 & $* * *$ & \\
\hline $\mathrm{IMC}$ & $<--$ & $\mathrm{VO}_{2} \mathrm{Max}$ & $-1,993$ & 0,185 & $-10,758$ & $* * *$ & $-0,577$ \\
\hline IMC & $<--$ & Salto & $-0,084$ & 0,008 & $-10,717$ & $* * *$ & $-0,433$ \\
\hline NIVELPAQC & $<--$ & IMC & $-0,161$ & 0,007 & $-23,198$ & $* * *$ & $-0,715$ \\
\hline HorasAF & $<--$ & IMC & $-0,148$ & 0,014 & $-10,571$ & $* * *$ & $-0,484$ \\
\hline HorasAF & $<--$ & NIVELPAQC & 0,343 & 0,062 & 5,541 & $* * *$ & 0,254 \\
\hline Autoestima & $<--$ & IMC & $-0,829$ & 0,068 & $-12,143$ & $* * *$ & $-0,368$ \\
\hline Autoestima & $<--$ & HorasAF & 0,941 & 0,195 & 4,815 & $* * *$ & 0,128 \\
\hline Autoestima & $<--$ & NIVELPAQC & 5,024 & 0,283 & 17,783 & $* * *$ & 0,503 \\
\hline Fuerza (DINA) & $<-->$ & Salto & 81,344 & 7,198 & 11,301 & $* * *$ & 0,575 \\
\hline $\mathrm{VO}_{2} \mathrm{Max}$ & $<-->$ & Salto & 15,217 & 1,082 & 14,062 & $* * *$ & 0,791 \\
\hline Fuerza (DINA) & $<-->$ & $\mathrm{VO}_{2} \mathrm{Max}$ & 6,245 & 0,445 & 14,026 & $* * *$ & 0,787 \\
\hline
\end{tabular}

PR: Pesos de regresión; PER: Pesos estandarizados de regresión; SE: Estimación del error; CR: Ratio crítico.

menor capacidad de salto. Además, el consumo de oxígeno es el predictor con mayor efecto $(-0,577)$ seguida de la potencia del salto $(-0,433)$. Por el contrario, la dinamometría manual, referida a la fuerza de prensión manual, muestra una relación directa y positiva con el IMC, aunque el peso de regresión es bastante bajo. Además, las tres variables predictoras se correlacionan significativamente de forma directa y positiva $(0,791 ; 0,575 ; 0,787)$.

En el segundo nivel del modelo podemos observar una relación directa y negativa entre el IMC y el nivel de AF (NIVELPAQC), las horas dedicadas a la actividad deportiva y el nivel de autoestima. Debemos destacar el peso de regresión asociado al nivel de $\mathrm{AF}(-0,715)$, revelando un aumento del IMC cuando disminuye la práctica de AF. Se observa una relación similar entre el IMC y las horas de $\mathrm{AF}(-0,484)$ y el nivel de autoestima en los adolescentes $(-0,368)$. Esta última relación revela que un IMC alto se asocia a niveles bajos de autoestima, explicando $81 \%$ de la varianza. Aunque tampoco podemos obviar el $51 \%$ de la varianza explicada entre el IMC y la $\mathrm{AF}$.

Finalmente, el nivel de AF (NIVELPAQC) se relaciona directa y positivamente $(0,503)$ con la autoestima, aunque la relación indirecta a través del número de horas dedicadas a la $\mathrm{AF}$ es menor $\left(0,03=0,254^{\star} 0,128\right)$. Estos valores muestran que la relación entre el nivel de AF y la autoestima de manera directa es mayor que de manera indirecta, a través de las horas dedicadas a la actividad deportiva (Tabla 1).

La evaluación del ajuste del modelo reveló una bondad de ajuste adecuada en la mayoría de los índices. El chi-cuadrado presenta un valor asociado de $\mathrm{p}$ significativo $\left(\chi^{2}=315,419 ; \mathrm{gl}=9\right.$; $\mathrm{p}=0,001)$, aunque debemos de tener en cuenta que este estadístico, como índice, no tiene límite superior. De este modo, no puede interpretarse de manera estandarizada, además del problema que plantea su sensibilidad al tamaño muestral. Por ello, se emplean otros índices de ajuste estandarizados que son menos sensibles al tamaño de la muestra $^{24}$. El índice comparativo de ajuste (CFI) que presenta un valor de 0,911 indica un ajuste aceptable ${ }^{25}$. El valor del índice de incremento de ajuste (IFI) también obtiene valores aceptables $(0,912)^{26}$. El índice de ajuste normalizado (NFI) obtiene valores superiores a 0,909 lo que también indica un ajuste aceptable. Por último, el valor de RMSEA es inferior a 0,08 , lo que indica un ajuste aceptable $(0,068)$ a los parámetros establecidos ${ }^{27}$. En suma, se observa un buen ajuste del modelo a los datos empíricos. 


\section{Discusión}

Este estudio tuvo como objetivo contrastar un modelo de ecuaciones estructurales que asocie el nivel de obesidad, la condición física, la práctica de AF y el nivel de autoestima; obteniéndose un buen ajuste para el modelo. De hecho, la importancia de esta línea de investigación ha sido puesta en alza por trabajos de revisión que analizan estudios similares en Reino Unido ${ }^{28}$, Chile ${ }^{18}$ o en América Latina $^{29}$, describiendo el estado del sobrepeso como problemática, factores de riesgo y medidas de prevención.

Los datos señalan que una menor capacidad de salto y de $\mathrm{VO}_{2}$ Max se asocia a un IMC más elevado. Estas cifras se encuentran en la misma línea que lo planteado por otros estudios ${ }^{30,31}$, los cuales revelan que aquellos niños con mayor capacidad aeróbica presentan menores niveles de grasa central; $y$ así lo demuestran las correlaciones directas y positivas entre las variables predictoras del modelo ( $\mathrm{VO}_{2} \mathrm{Max}$, dinamometría y salto). Estas tres capacidades determinan valores similares en la muestra de estudio, estableciéndose una clara asociación entre el estado de peso corporal y la condición física.

En referencia al IMC, los datos nos muestran una relación inversa con el nivel de AF y de autoestima. Estos resultados confirman tanto los efectos beneficiosos de la práctica físico-deportiva en el estado de salud de los escolares, como la influencia negativa de un IMC elevado en diversas dimensiones psicosociales vinculadas a la calidad de vida y el estado de salud. Varios trabajos de investigación plasman resultados similares, indicando la posibilidad de estas asociaciones fuesen propiciadas por el nivel socioeconómico de la población de estudio ${ }^{28,32}$. Asimismo, los valores obtenidos son similares a los de estudios recientes, donde se pone de manifiesto que la AF está positivamente relacionada con la salud, principalmente en los aspectos mentales, sociales y físicos de las personas ${ }^{32,33}$.

Es el área de Educación Física desde donde el docente debe evaluar la condición física de sus alumnos y observar su evolución a lo largo del período de escolarización, siendo necesario realizar registros del estado de salud y la condición física de los escolares para evitar la alta prevalencia de obesidad alcanzada en Chile, cuya causa se asocia a la impresionante velocidad de la transición nu- tricional ${ }^{34,35}$. De hecho, existen datos generales que indican que alrededor de un tercio de todos los niños son obesos a nivel global, haciendo pensar que los niños obesos serán adultos obesos ${ }^{32,34}$.

Asimismo, se observó una relación positiva y directa entre el nivel de AF y el de autoestima. En este sentido, debemos indicar como uno de los beneficios a nivel psicosocial que tiene la práctica deportiva regular en edades tempranas, la mejora de la propia percepción del niño. Para finalizar seguimos las premisas planteadas en diversos estudios, las cuales sugieren que la AF puede desempeñar un papel protagonista en la prevención de la obesidad en niños y adolescentes ${ }^{36}$, así como una mejora de la percepción que tiene un individuo de sí mismo.

Puede concluirse que el IMC se relaciona de forma negativa con la capacidad aeróbica, la capacidad de salto y los niveles de autoestima, así como con las horas de práctica de AF. Igualmente se detecta una relación positiva entre el nivel de AF y el nivel de autoestima de los escolares.

Las repercusiones prácticas que puede tener este estudio son amplias, aportando aspectos que tienen que ver con la disminución de los índices de obesidad para mejorar parámetros psicosociales y físico-deportivos. Al hilo de los hallazgos de este trabajo, sería de interés realizar intervenciones encaminadas al aumento de hábitos físico-saludables para la mejora del IMC, la condición física y la autoestima en escolares, estableciendo relaciones causa-efecto.

Agradecimientos: A Vicerrectoría de Investigación y Desarrollo de la Universidad de Santiago de Chile. Proyectos Basales.

\section{Referencias}

1. Ministerio de Salud (2011). Estrategia nacional de salud, para el cumplimiento de los objetivos sanitarios de la década 2011-2020. Extraído de: http://web.minsal.cl/ portal/url/item/c4034eddbc96ca6de0400101640159b8. pdf.

2. Finkelstein EA, Fiebelkorn IC, Wang G. National medical spending attributable to overweight and obesity: how much, and who's paying? Health Affairs 2003; 22 (3): 213-9.

3. Lobos LL, Leyton B, Kain J, Vio, F. Evaluación de una intervención educativa para la prevención de la obesidad 
infantil en escuelas básicas de Chile. Nutr Hosp 2013; 28 (4): 1156-64.

4. Santos JL, Ho-Urriola JA, González A, Smalley SV, Domínguez-Vásquez P, Cataldo R, et al. Association between eating behavior scores and obesity in Chilean children. Nutr J 2011; 10 (1): 1-8.

5. Haskell WL, Lee IM, Pate RR, Powell KE, Blair SN, Franklin BA. Physical activity and public health: updated recommendation for adults from the American College of Sports Medicine and the American Heart Association. Circulation 2007; 116 (9): 1081-93.

6. Janssen I, LeBlanc AG. Systematic review of the health benefits of physical activity and fitness in school-aged children and youth. Int J Behav Nutr Phys Act 2010; 7 (40): 1-16.

7. McGinnis JM, Williams-Russo P, Knickman JR. The case for more active policy attention to health promotion. Health Affairs 2002; 21 (2): 78-93.

8. Ostojic S, Stojanovic MD, Stojanovic V, Maric J, Njaradi N. Correlation between Fitness and Fatness in 6-14-year Old Serbian School Children. J Health Popul Nutr 2011; 29 (1): 53-60.

9. Cuenca-García M, Jiménez-Pavón D, España-Romero V, Artero E, Castro-Piñero J, Ortega F, et al. Condición física relacionada con la salud y hábitos de alimentación en niños y adolescentes: propuesta de addendum al informe de salud escolar. Rev Inv Educ 2011; 9 (2): 35-50.

10. Steene-Johannessen J, Anderssen SA, Kolle E, Andersen LB. Low muscle fitness is associated with metabolic risk in youth. Med \& Sci Sport \& Exerc 2009; 41 (7): 1361-7.

11. Kemper HC, De Vente W, Van Mechelen W, Twisk JW. Adolescent motor skill and performance: is physical activity in adolescence related to adult physical fitness? Am J Human Biology 2001; 13 (2): 180-9.

12. Naranjo, CR, González AC. Autoestima en la adolescencia: análisis y estrategias de intervención. Int J Psychol Psychol Ther 2012; 12 (3): 389-403.

13. Kuhlberg JA, Peña JB, Zayas LH. Familism, parent-adolescent conflict, self-esteem, internalizing behaviors and suicide attemps among adolescent Latinas. Child Psych Hum Develop 2010; 41 (4): 425-40.

14. Ybrandt H, Armelius K. Peer aggression and mental health problems self-esteem as a mediator. School Psychol Int 2010; 31 (2): 146-63.

15. Donnellan MB, Trzesniewski KH, Robins RW, Moffitt TE, Caspi A. Low self-esteem is related to aggression, antisocial behavior, and delinquency. Psychol Sci 2005; $16(4): 328-35$.

16. Martínez-Maldonado R, Pedrão LJ, Alonso-Castillo MM, López-García KS, Oliva-Rodríguez NN. Autoesti- ma, autoeficacia percibida, consumo de tabaco y alcohol en estudiantes de educación secundaria de área urbana y rural de Monterrey, Nuevo León, México. Rev Lat Am Enfermagem 2008; 16 (1): 614-20.

17. Trzesniewski KH, Donnellan MB, Moffitt TE, Robins RW, Poulton R. Caspi A. Low self-esteem during adolescence predicts poor health, criminal behavior, and limited economic prospects during adulthood. Develop Psychol 2006; 42 (2): 381-90.

18. Kain J, Lera L, Rojas J, Uauy R. Obesidad en preescolares de la Región Metropolitana de Chile. Rev Med Chile 2007; 135 (1): 63-70.

19. Vio F, Albala C, Kain J. Nutrition transition in Chile revisited: mid-term evaluation of obesity goals for the period 2000-2010. Public Health Nutr 2008; 11 (4): 405-12.

20. Davis SP, Northington L, Kolar K. Cultural considerations for treatment of childhood obesity. J Cult Diversity 2000; 7 (4): 128.

21. Rosemberg M. La autoimagen del adolescente y la sociedad. Buenos Aires (Argentina): Paidos; 1965.

22. Martín-Albo J, Núñez JL, Navarro JG, Grijalvo F. The Rosenberg Self-Esteem Scale: Translation and Validation in University Students. The Spanish Journal of Psychology 2007; 10 (2): 458-69.

23. Kowalski KC, Crocker PR, Donen RM. The physical activity questionnaire for older children (PAQ-C) and adolescents (PAQ-A) manual. College of Kinesiology, University of Saskatchewan 2004; 87(1): 1-38.

24. Jöreskog KG. Structural Equation Models in the Social Sciences: Specification estimation and testing. En P.R. Krishnaiah (Ed.), Applications of Statistics, pp. 265-287. Amsterdam: North Holland, 1997.

25. Hu LT, Bentler PM. Fit indices in covariance structure model-ing: sensitivity to under parameterized model misspecification. Psychological Methods 1998; 3: 42453.

26. Bollen KA. Structural Equations with Latent Variables. New York: John Wiley y Sons, Inc; 1989.

27. Browne MW, Cudeck R. Alternative ways of assessing model fit. En: K. A. Bollen y J. S. Long (Eds.), Testing structural equation models (pp. 136-162). Beverly Hills, CA: Sage; 1993.

28. Riazi A, Shakoor S, Dundas I, Eiser C, Mckenzie S. Health-related quality of life in a clinical sample of obese children and adolescents. Health Qual Life Outcomes 2010; 8 (1): 134-134.

29. Rivera J, Cossío T, Pedraza L, Aburto T, Sánchez T, Martorell R. Childhood and adolescent overweight and obesity in Latin America: A systematic review. The Lancet Diabetes \& Endocrinology 2014; 321-32. 
30. Ara I, Vicente-Rodríguez G, Moreno LA, Gutin B, Casajus JA. La obesidad infantil se puede reducir mejor mediante actividad física vigorosa que mediante restricción calórica. Apunts. Medicina de l'Esport 2009; 44 (163): 111-8.

31. Winsley RJ, Armstrong N, Middlebrooke AR, Ramos-Ibáñez N, Williams CA. Aerobic fitness and visceral adipose tissue in children. Acta Pediátrica 2006; 95 (11): 1435-8.

32. Silva JR, Capurro G, Saumann MP, Slachevsky A. Problematic eating behaviors and nutritional status in 7 to 12 year-old Chilean children. Int J Clinical Health Psychol 2013; 13 (1): 32-9.

33. Trost SG, Kerr LM, Ward DS, Pate RR. Physical activity and determinants of physical activity in obese and non-obese children. International journal of obesity and related metabolic disorders. J Int Association Study Obesity 2001; 25 (6): 822-9.

34. Cuenca-García M, Jiménez-Pavón D, España-Romero V, Artero E, Castro-Piñero J, Ortega FB, et al. Condición física relacionada con la salud y hábitos de alimentación en niños y adolescentes: Propuesta de addendum al informe de salud Escolar. Rev Inv Educ 2011; 9 (2): 35-50.

35. Zayas GM, Chiong D, Díaz Y, Torriente A, Herrera X. Obesidad en la infancia: Diagnóstico y tratamiento. Rev Cubana Pediatría 2002; 74: 1-15.

36. Gutin B, Yin Z, Humphries MC, Barbeau P. Relations of moderate and vigorous physical activity to fitness and fatness in adolescents. Am J Clinical Nutr 2005; 81 (4): 746-50. 